TRANSACTIONS OF THE

AMERICAN MATHEMATICAL SOCIETY

Volume 358, Number 2, Pages 799-818

S 0002-9947(05)03723-2

Article electronically published on May 10, 2005

\title{
LAGRANGIAN SUBMANIFOLDS AND MOMENT CONVEXITY
}

\author{
BERNHARD KRÖTZ AND MICHAEL OTTO
}

\begin{abstract}
We consider a Hamiltonian torus action $T \times M \rightarrow M$ on a compact connected symplectic manifold $M$ and its associated momentum map $\Phi$. For certain Lagrangian submanifolds $Q \subseteq M$ we show that $\Phi(Q)$ is convex. The submanifolds $Q$ arise as the fixed point set of an involutive diffeomorphism $\tau: M \rightarrow M$ which satisfies several compatibility conditions with the torus action, but which is in general not anti-symplectic. As an application we complete a symplectic proof of Kostant's non-linear convexity theorem.
\end{abstract}

\section{INTRODUCTION}

In the context of Hamiltonian torus actions $T \times M \rightarrow M$ on a connected symplectic manifold $M$ one is interested in convexity properties of the image of the associated momentum map $\Phi: M \rightarrow \mathfrak{t}^{*}$. This is because of its many applications to classical eigenvalue problems and their Lie theoretic generalizations. In this paper we will determine a class of Lagrangian submanifolds $Q \subseteq M$ for which $\Phi(Q)$ is convex. Applications to a symplectic proof of Kostant's non-linear convexity theorem will be given.

Before we will describe our results in more detail, it is useful to summarize some known convexity results for the momentum map $\Phi$. We recall that the critical set of $\Phi$ is the set $\operatorname{Fix}(M)$ of $T$-fixed points in $M$. Then the convexity theorem of Atiyah-Guillemin-Sternberg [2, 5] reads as follows.

Theorem 1.1. If $M$ is compact, then $\Phi(M)$ is convex. More precisely, $\Phi(M)$ is the convex polyhedron spanned by the finite set $\Phi(\operatorname{Fix}(M))$.

This theorem has been generalized by Duistermaat [4]. Assume that $M$ carries an anti-symplectic involution $\tau: M \rightarrow M$ and write $Q$ for the fixed point set of $\tau$. We require that $Q$ is non-empty. Then $Q$ is a Lagrangian submanifold of $M$, and Duistermaat's convexity theorem [4, Th. 2.5] says:

Theorem 1.2. If $M$ is compact, and $\tau: M \rightarrow M$ is an anti-symplectic involution which satisfies $\Phi \circ \tau=\Phi$, then $\Phi(Q)=\Phi(M)$. In particular $\Phi(Q)$ is convex.

Duistermaat's theorem was further generalized to the case where $M$ is noncompact and the momentum map proper [9], 14]. But even if $\Phi$ is not proper, there are interesting classes of symplectic manifolds for which $\Phi(M)$ is still convex (cf. [7], [10], 14]).

Received by the editors November 11, 2003 and, in revised form, March 31, 2004.

2000 Mathematics Subject Classification. Primary 53D20, 22E15.

The work of the first author was supported in part by NSF grant DMS-0097314.

(C)2005 American Mathematical Society 
In this paper we want to give another generalization of Theorem 1.2 which goes in a different direction. It turns out that the assumption that the involution $\tau$ is anti-symplectic is too strong for certain applications. However, we will show that under relaxed conditions on $\tau$ we can still derive the results of Duistermaat's theorem. In particular,

Theorem 3.1. Let $M$ be a compact connected symplectic manifold with Hamiltonian torus action $T \times M \rightarrow M$ and momentum map $\Phi: M \rightarrow \mathfrak{t}^{*}$. In addition, let $\tau: M \rightarrow M$ be an involutive diffeomorphism with fixed point set $Q$ such that

1. $t \circ \tau=\tau \circ t^{-1}$ for all $t \in T$.

2. $\Phi \circ \tau=\Phi$.

3. $Q$ is a Lagrangian submanifold of $M$.

Then $\Phi(Q)=\Phi(M)$. In particular $\Phi(Q)$ is a convex subset of $\mathfrak{t}^{*}$. Moreover, the same assertions hold if $Q$ is replaced with any of its connected components.

As our main application of Theorem 3.1 we will complete the symplectic proof of Kostant's non-linear convexity theorem as given in [13.

Let us briefly recall Kostant's result. Let $G=N A K$ be an Iwasawa decomposition of a semisimple linear Lie group $G$. Write $\widetilde{a}: G \rightarrow A$ for the associated middle projection. The Lie algebras of $G, N, A$ and $K$ shall be denoted by $\mathfrak{g}, \mathfrak{n}, \mathfrak{a}$ and $\mathfrak{k}$. Then Kostant's Theorem [11] asserts that

$$
(\forall X \in \mathfrak{a}) \quad \log \widetilde{a}(K \exp (X))=\operatorname{conv}(\mathcal{W} \cdot X),
$$

where $\operatorname{conv}(\mathcal{W} . X) \subseteq \mathfrak{a}$ denotes the convex hull of the Weyl group orbit $\mathcal{W} . X$.

$\mathrm{Lu}$ and Ratiu [13] were able to deduce Kostant's result from the AGS-convexity theorem for a complex group $G$.

If $G$ is not complex the situation is different. For those groups for which $\mathfrak{m}=\mathfrak{z k}(\mathfrak{a})$ is abelian one can show (1.1) using Duistermaat's theorem [13], since in these cases the involution $\tau$ one encounters is indeed anti-symplectic 8 .

If $\mathfrak{m}$ is not abelian, $\tau$ still satisfies the assumptions 1.-3. in Theorem 3.1. Therefore, Theorem 3.1 can be used to give a symplectic proof of Kostant's theorem for an arbitrary $G$.

It is our pleasure to thank Robert J. Stanton for his very useful advice on structure and presentation of the underlying paper. We would also like to thank the referee for his careful work.

\section{LOCAL RESULTS}

This section lays the foundation for the proof of the convexity theorem in Chapter 3. We will give local descriptions for the momentum map $\Phi$ and its restriction $\left.\Phi\right|_{Q}$ in Subsection 2.2. In 2.1, we fix the notation and prove a lemma on the characterization of anti-symplectic involutions on a symplectic vector space which is needed in 2.2 .

2.1. Background. Let $(M, \omega)$ denote a connected symplectic manifold with $\operatorname{dim} M=2 n$. Then every smooth function $f \in C^{\infty}(M)$ determines a Hamiltonian vector field $\mathcal{X}_{f}$ on $M$ which is defined by $d f=i\left(\mathcal{X}_{f}\right) \omega$. From $\omega$ one obtains the usual Poisson structure on $M$ :

$$
\{\cdot, \cdot\}: C^{\infty}(M) \times C^{\infty}(M) \rightarrow C^{\infty}(M), \quad(f, g) \mapsto\{f, g\}=\omega\left(\mathcal{X}_{f}, \mathcal{X}_{g}\right) .
$$


Our next datum is a torus $T$ which we require to act symplectically on $M$. Write

$$
T \times M \rightarrow M, \quad(t, m) \mapsto t . m
$$

for this action.

Let $\mathfrak{t}$ denote the Lie algebra of $T$. For $X \in \mathfrak{t}$ let $\widetilde{X}$ be the corresponding vector field on $M$, i.e.,

$$
\widetilde{X}_{m}=\left.\frac{d}{d t}\right|_{t=0} \exp (t X) \cdot m \quad(m \in M) .
$$

We will always assume that the action of $T$ on $M$ is Hamiltonian, that is, there exists a $T$-equivariant Lie algebra homomorphism

$$
\mathfrak{t} \rightarrow\left(C^{\infty}(M),\{\cdot, \cdot\}\right), \quad X \mapsto \Phi_{X}
$$

such that

$$
i(\widetilde{X}) \omega=d \Phi_{X}
$$

holds for all $X \in \mathfrak{t}$. If $\mathfrak{t}^{*}$ denotes the dual of $\mathfrak{t}$, then the assignment

$$
\Phi: M \rightarrow \mathfrak{t}^{*} ; \quad\langle\Phi(m), X\rangle=\Phi_{X}(m) \quad(m \in M, X \in \mathfrak{t})
$$

defines a smooth map, called the momentum map.

Let $\tau: M \rightarrow M$ be an involutive diffeomorphism. We will denote by $Q$ its fixed point set, i.e.

$$
Q=\{m \in M: \tau(m)=m\},
$$

and require $Q$ to be non-empty. Notice that $Q$ is a closed submanifold of $M$. If in addition $\tau$ is anti-symplectic, then $Q$ is a Lagrangian submanifold of $M$.

Write $\operatorname{Fix}(M)$ for the set of $T$-fixed points in $M$. Equivalently $\operatorname{Fix}(M)$ is the critical set of the momentum map $\Phi: M \rightarrow \mathfrak{t}^{*}$.

We will be interested in involutions $\tau$ satisfying the following conditions:

$$
\begin{aligned}
& t \circ \tau=\tau \circ t^{-1} \text { for all } t \in T, \\
& \Phi \circ \tau=\Phi, \\
& Q \text { is a Lagrangian submanifold of } M .
\end{aligned}
$$

Some remarks on (2.1.2)-(2.1.4) are appropriate.

Remark 2.1.1. (a) Notice that we do not assume that the involution $\tau$ is antisymplectic; however we require the fixed point manifold $Q$ to be Lagrangian.

(b) The conditions in Duistermaat's theorem are stronger than (2.1.2)-(2.1.4). In fact, if $\tau$ is anti-symplectic, then (2.1.2) and (2.1.3) are equivalent.

Note that condition (2.1.2) is equivalent to

$$
d \tau(m) \widetilde{X}_{m}=-\widetilde{X}_{\tau(m)} \quad(m \in M, X \in \mathfrak{t}) .
$$

In particular we have

$$
d \tau(m) \widetilde{X}_{m}=-\widetilde{X}_{m} \quad(m \in Q, X \in \mathfrak{t}) .
$$

First we shall investigate conditions (2.1.2) and (2.1.4) in the linear case, i.e. when $(M, \omega)$ is a symplectic vector space with linear torus action and linear involution $\tau$.

Let $(V, \Omega)$ denote a finite-dimensional symplectic vector space and $\tau: V \rightarrow V$ a linear involution. As $\tau \circ \tau=\mathrm{id}_{V}$, the linear operator $\tau$ is semisimple with eigenvalues +1 and -1 . Accordingly we have an eigenspace decomposition $V=V_{1} \oplus V_{-1}$. 
Next we endow $(V, \Omega)$ with a linear symplectic torus action $T \times V \rightarrow V$. Then $V$ decomposes into fixed and effective part

$$
V=V_{\text {fix }} \oplus V_{\text {eff }}
$$

where

$$
\begin{aligned}
V_{\text {fix }} & =\{v \in V:(\forall X \in \mathfrak{t}) X . v=0\} \\
& =\{v \in V:(\forall t \in T) t . v=v\}
\end{aligned}
$$

and

$$
V_{\text {eff }}=\mathfrak{t} . V \text {. }
$$

Notice that $\Omega$ is non-degenerate when restricted to $V_{\text {fix }}$ or $V_{\text {eff }}$. Hence both $V_{\text {fix }}$ and $V_{\text {eff }}$ become symplectic subspaces of $V$.

The following lemma might be known to many; different versions of it are frequently encountered in the literature. Nevertheless we wish to provide its simple proof. Note that the first three statements do not rely on the existence of the torus action at all.

Lemma 2.1.2. Let $(V, \Omega)$ be a symplectic vector space and let $\tau: V \rightarrow V$ be a linear involution with eigenspace decomposition $V=V_{1} \oplus V_{-1}$. Then the following statements are equivalent:

1. $\tau$ is anti-symplectic.

2. $V_{1}$ and $V_{-1}$ are Lagrangian.

3. $V_{1}$ is Lagrangian and there exists a symplectic isomorphism $\varphi: V \rightarrow V$ with $\varphi\left(V_{1}\right)=V_{-1}$ and $\varphi\left(V_{-1}\right)=V_{1}$.

4. $V_{1}$ is Lagrangian and there exists a linear symplectic torus action $T \times V \rightarrow V$ with the following properties:

a. $t \circ \tau=\tau \circ t^{-1} \quad \forall t \in T$.

b. $V_{\text {fix }}=\{0\}$.

Proof. For $v \in V$ let $v_{1} \in V_{1}, v_{-1} \in V_{-1}$ be such that $v=v_{1}+v_{-1}$.

1. $\Longrightarrow 2 .:$ Assume that $\tau$ is anti-symplectic. Then

$$
\Omega\left(v_{1}, w_{1}\right)=\Omega\left(\tau\left(v_{1}\right), \tau\left(w_{1}\right)\right)=-\Omega\left(v_{1}, w_{1}\right),
$$

and similarly,

$$
\Omega\left(v_{-1}, w_{-1}\right)=\Omega\left(-\tau\left(v_{-1}\right),-\tau\left(w_{-1}\right)\right)=-\Omega\left(v_{-1}, w_{-1}\right) .
$$

This implies that $V_{1}$ and $V_{-1}$ are isotropic. As $V=V_{1} \oplus V_{-1}$, it follows that both $V_{1}$ and $V_{-1}$ are maximally isotropic, i.e. Lagrangian subspaces of $V$.

2. $\Longrightarrow 1 .:$ Assume that $V_{1}$ and $V_{-1}$ are Lagrangian. We compute

$$
\begin{aligned}
\Omega\left(v_{1}+v_{-1}, w_{1}+w_{-1}\right) & =\Omega\left(v_{1}, w_{1}\right)+\Omega\left(v_{-1}, w_{1}\right)+\Omega\left(v_{1}, w_{-1}\right)+\Omega\left(v_{-1}, w_{-1}\right) \\
& =\Omega\left(v_{-1}, w_{1}\right)+\Omega\left(v_{1}, w_{-1}\right)
\end{aligned}
$$

and

$$
\begin{aligned}
\Omega\left(\tau\left(v_{1}+v_{-1}\right), \tau\left(w_{1}\right.\right. & \left.\left.+w_{-1}\right)\right)=\Omega\left(v_{1}-v_{-1}, w_{1}-w_{-1}\right) \\
& =\Omega\left(v_{1}, w_{1}\right)-\Omega\left(v_{-1}, w_{1}\right)-\Omega\left(v_{1}, w_{-1}\right)+\Omega\left(v_{-1}, w_{-1}\right) \\
& =-\Omega\left(v_{1}+v_{-1}, w_{1}+w_{-1}\right) .
\end{aligned}
$$

Hence, $\tau$ is anti-symplectic.

2. $\Longrightarrow 3 .:$ As Lagrangian subspaces both $V_{1}$ and $V_{-1}$ have dimension $n=$ $\frac{1}{2} \operatorname{dim} V$. Let $\left\{e_{1}, \ldots, e_{n}\right\}$ be a basis of $V_{1}$. As $\Omega$ is non-degenerate, assumption 2 . 
implies that there is a basis $\left\{f_{1}, \ldots, f_{n}\right\}$ of $V_{-1}$ such that $\Omega\left(e_{i}, f_{j}\right)=\delta_{i j}$ for all $1 \leq i, j \leq n$.

Define $\varphi$ by

$$
\varphi\left(e_{i}\right)=f_{i}, \quad \varphi\left(f_{i}\right)=-e_{i} \quad \forall i .
$$

Then for all $1 \leq i, j \leq n$

$$
\Omega\left(e_{i}, f_{j}\right)=\delta_{i j}=\Omega\left(e_{j}, f_{i}\right)=-\Omega\left(f_{i}, e_{j}\right)=\Omega\left(\varphi\left(e_{i}\right), \varphi\left(f_{j}\right)\right),
$$

completing the proof of $1 . \Longrightarrow 3$.

$3 . \Longrightarrow 2 .:$ It suffices to show that $V_{-1}$ is isotropic. But this follows from the fact that $\varphi$ is surjective and that for $v, w \in V_{1}$,

$$
\Omega(\varphi(v), \varphi(w))=\Omega(v, w)=0 .
$$

3. $\Longrightarrow 4$.: For $\mathfrak{t}=\mathbb{R} \varphi$, the corresponding torus action by $T=\exp \mathfrak{t}$ clearly has the desired properties.

4. $\Longrightarrow 2 .:$ We have to show that $V_{-1}$ is Lagrangian. Let $T \times V \rightarrow V$ be a torus action which satisfies a. and $b$. We notice that $a$. is equivalent to its infinitesimal version

$$
\tau \circ X=-X \circ \tau \quad(X \in \mathfrak{t}) .
$$

Let $X \in \mathfrak{t}$. Then it is immediate from (2.1.7) that

$$
X\left(V_{1}\right) \subseteq V_{-1} \quad \text { and } \quad X\left(V_{-1}\right) \subseteq V_{1} .
$$

It follows from b. that there is an element $Y \in \mathfrak{t}$ such that $Y: V \rightarrow V$ is invertible. Hence (2.1.8) implies that

$$
Y\left(V_{1}\right)=V_{-1} \quad \text { and } \quad Y\left(V_{-1}\right)=V_{1} .
$$

Let $v_{-1}, w_{-1} \in V_{-1}$. By (2.1.9) we find $v_{1}, w_{1} \in V_{1}$ such that $Y \cdot v_{1}=v_{-1}$ and $Y . w_{1}=w_{-1}$. As $V_{1}$ is Lagrangian, $Y^{2}\left(V_{1}\right)=V_{1}$ (by (2.1.9)) and $\Omega$ is $T$-invariant, it now follows that

$$
\Omega\left(v_{-1}, w_{-1}\right)=\Omega\left(Y \cdot v_{1}, Y \cdot w_{1}\right)=-\Omega\left(Y^{2} \cdot v_{1}, w_{1}\right)=0 .
$$

Hence $V_{-1}$ is Lagrangian.

2.2. Local normal forms. Throughout this subsection we will assume that $(M, \omega)$ is a connected symplectic manifold endowed with a Hamiltonian torus action with momentum map $\Phi$. Also, we have an involution $\tau$ which satisfies (2.1.2)(2.1.4) and whose fixed point set we denote by $Q$. Our objective is to provide a local normal form for $\left.\Phi\right|_{Q}$ near a point $m \in Q$. To that end we first recall a method of finding suitable local descriptions for $\omega$ and $\Phi$ in the neighborhood of a generic point $m \in M$. We then consider points $m \in Q$ and obtain a refined form of $\left.\Phi\right|_{Q}$ which is adapted to the involution $\tau$. We start with a simple observation (cf. 4, Lemma 2.1]):

Lemma 2.2.1. Let $m \in Q$ and $X \in \mathfrak{t}$. Then $d\left(\left.\Phi_{X}\right|_{Q}\right)(m)=0$ implies $d \Phi_{X}(m)=$ 0 . In particular, $m$ is fixed under the action of the one parameter subgroup $\exp (\mathbb{R} X)$.

Proof. Write $E=\mathcal{T}_{m} M$ for the tangent space at $m$. Let $E=E_{1} \oplus E_{-1}$ be the decomposition of $E$ into \pm 1-eigenspaces of the involution $d \tau(m)$. Notice that $\mathcal{T}_{m} Q=$ $E_{1}$. In order to show that $d \Phi_{X}(m)=0$ it hence suffices to prove $d \Phi_{X}(m)(v)=0$ for all $v \in E_{-1}$. 
Let $v \in E_{-1}$. Then it follows from (2.1.3) that

$$
d \Phi_{X}(m)(v)=-d \Phi_{X}(m)(d \tau(m) v)=-d\left(\Phi_{X} \circ \tau\right)(m)(v)=-d \Phi_{X}(m)(v),
$$

and so $d \Phi_{X}(m)(v)=0$. The last assertion in the lemma follows from (2.1.1). This concludes the proof of the lemma.

For $m \in M$ we write $T_{m}$ for the stabilizer of $T$ in $m$, i.e.

$$
T_{m}=\{t \in T: t . m=m\} \text {. }
$$

The Lie algebra $\mathfrak{t}_{m}$ of $T_{m}$ is then given by

$$
\mathfrak{t}_{m}=\left\{X \in \mathfrak{t}: \widetilde{X}_{m}=0\right\} .
$$

If in addition $m \in Q$, then it follows from Lemma 2.2.1 that we can equally characterize $\mathfrak{t}_{m}$ by

$$
\mathfrak{t}_{m}=\left\{X \in \mathfrak{t}: d\left(\left.\Phi_{X}\right|_{Q}\right)(m)=0\right\} .
$$

Fix now $m \in M$. Next we provide local normal forms for $\omega$ and $\Phi$ near $m$. We will recall the procedure of momentum reconstruction (cf. [6, Ch. 41]): The momentum map near $m$ is uniquely characterized by $\Phi(m)$, the stabilizer $T_{m}$ and the linear representation of $T_{m}$ on the tangent space $\mathcal{T}_{m} M$. This is even true for a general compact Lie group $T$ and in the case of a torus was further exploited in 9 , Sect. 2].

Notice that $\mathcal{T}_{m}($ T.m $)$ is an isotropic subspace of $\mathcal{T}_{m} M$. Thus $\omega_{m}$ induces on the quotient

$$
V=\mathcal{T}_{m}(T . m)^{\perp} / \mathcal{T}_{m}(T . m)
$$

a symplectic form $\Omega$. We write $\operatorname{Sp}(V, \Omega)$ for the corresponding symplectic group. Notice that the isotropy subgroup $T_{m}$ acts on $\mathcal{T}_{m} M$ symplectically. Clearly this action leaves $\mathcal{T}_{m}($ T.m $)$ and hence $\mathcal{T}_{m}(T . m)^{\perp}$ invariant, thus giving rise to a representation on $V$, say

$$
\pi: T_{m} \rightarrow \operatorname{Sp}(V, \Omega)
$$

Write $\left(T_{m}\right)_{0}$ for the connected component of $T_{m}$ containing 1 . Notice that $\left(T_{m}\right)_{0}<T$ is a subtorus and so we can find a torus complement $S_{m}$ to $\left(T_{m}\right)_{0}$ in $T$, i.e.

$$
T=S_{m} \times\left(T_{m}\right)_{0} .
$$

We denote the Lie algebra of $S_{m}$ by $\mathfrak{s}_{m}$. Then $\mathfrak{t}=\mathfrak{t}_{m} \oplus \mathfrak{s}_{m}$, and we have a canonical identification $\mathfrak{t}^{*}=\mathfrak{s}_{m}^{*} \times \mathfrak{t}_{m}^{*}$. Denote by $\mathcal{T}^{*} S_{m}$ the cotangent bundle of $S_{m}$ with its canonical symplectic structure. In the sequel we use the identification $\mathcal{T}^{*} S_{m}=\mathfrak{s}_{m}^{*} \times S_{m}$. Hence $\mathcal{T}^{*} S_{m} \times V$ carries a natural symplectic structure. Further $T=S_{m} \times\left(T_{m}\right)_{0}$ acts symplectically on $\mathcal{T}^{*} S_{m} \times V$ via

$$
(s, t) \cdot\left(\beta, s^{\prime} v\right):=\left(\beta, s s^{\prime}, \pi(t) v\right)
$$

for $s, s^{\prime} \in S_{m}, t \in\left(T_{m}\right)_{0}, \beta \in \mathfrak{s}_{m}^{*}$ and $v \in V$. It follows from [9. Lemma 2.1] that there is a symplectic diffeomorphism

$$
\rho: \mathcal{T}^{*} S_{m} \times V \supseteq \mathcal{U} \rightarrow U \subseteq M
$$

from an open neighborhood $\mathcal{U}$ of $(0,1,0) \in \mathcal{T}^{*} S_{m} \times V$ to an open neighborhood $U$ of $m$ such that $\rho$ is locally $T$-equivariant and satisfies $\rho(0, \mathbf{1}, 0)=m$.

In the following we will identify $U$ with $\mathcal{U} \subseteq \mathcal{T}^{*} S_{m} \times V$ via our symplectic, locally $T$-equivariant chart $\rho: \mathcal{U} \rightarrow U$. Write $V=V_{\text {fix }} \oplus V_{\text {eff }}$ for the decomposition of $V$ in 
the effective and fixed part for the linear action of $T_{m}$ on $V$ (cf. (2.1.6)). Furthermore we have the $\mathfrak{t}_{m}$-weight space decomposition $V=\bigoplus_{\lambda \in \Lambda} V_{\lambda}$. We decompose elements $v \in V$ as $v=\sum_{\lambda \in \Lambda} v_{\lambda}$ with $v_{\lambda} \in V_{\lambda}$. Recall that there is a $T_{m}$-invariant complex structure $J$ on $V_{\text {eff }}$ such that $\langle v, w\rangle=\Omega(v, J w)$ defines a positive definite scalar product on $V_{\text {eff. }}$ Then it follows from [9, Lemma 2.2] that the local normal form of $\Phi$ near a generic point $m \in M$ is given on $\mathcal{U}$ by

$$
\Phi: \mathcal{U} \rightarrow \mathfrak{t}^{*}=\mathfrak{s}_{m}^{*} \times \mathfrak{t}_{m}^{*}, \quad(\beta, s, v) \mapsto \Phi(0, \mathbf{1}, 0)+\left(\beta, \frac{1}{2} \sum_{\substack{\lambda \in \Lambda \\ \lambda \neq 0}}\left\|v_{\lambda}\right\|^{2} \lambda\right) .
$$

Assume now that $m \in Q$. So far we have not addressed the question of the nature of $Q$ and $\tau$ within our new coordinates in $\mathcal{T}^{*} S_{m} \times V$. In case $\tau$ is anti-symplectic on $M$, there is a beautiful answer, namely $\tau(\beta, s, v)=\left(\beta, s^{-1}, \tau_{V}(v)\right)$. However, with our restricted assumption (2.1.2)-(2.1.4) we cannot hope for such a nice form.

Near $(0, \mathbf{1}, 0)$ the shape of $Q$ is essentially determined by the linear involution $\sigma:=d \tau(0, \mathbf{1}, 0)$ on $E=\mathcal{T}_{(0, \mathbf{1}, 0)} \mathcal{U} \simeq \mathcal{T}_{m} M$.

We will use the natural identification $E=\mathfrak{s}_{m}^{*} \times \mathfrak{s}_{m} \times V$. Define $W=\mathfrak{s}_{\mathfrak{m}}^{*} \times \mathfrak{s}_{m} \times V_{\text {fix }}$. Then it follows from (2.2.2) that $E=W \oplus V_{\text {eff }}$ is the decomposition of $E$ into fixed and effective part of the isotropy action of $T_{m}$ on $E \simeq \mathcal{T}_{m} M$. Then (2.1.2) implies that the involution $\sigma$ leaves the decomposition $E=W \oplus V_{\text {eff }}$ invariant. Hence

$$
\sigma=\left(\begin{array}{cc}
\left.\sigma\right|_{W} & 0 \\
0 & \left.\sigma\right|_{V_{\text {eff }}}
\end{array}\right) .
$$

Accordingly we have a splitting

$$
\mathcal{T}_{(0, \mathbf{1}, 0)} Q=\left(\mathcal{T}_{(0, \mathbf{1}, 0)} Q \cap W\right) \oplus\left(\mathcal{T}_{(0, \mathbf{1}, 0)} Q \cap V_{\text {eff }}\right) .
$$

Next we will analyze the pieces $\left.\sigma\right|_{W}$ and $\left.\sigma\right|_{V_{\text {eff }}}$. We start with $\left.\sigma\right|_{V_{\text {eff }}}$. Notice that it follows from (2.1.2) that

$$
\left.\pi(t) \circ \sigma\right|_{V_{\text {eff }}}=\left.\sigma\right|_{V_{\text {eff }}} \circ \pi\left(t^{-1}\right) \quad\left(t \in T_{m}\right) .
$$

Thus we can apply Lemma 2.1.2 (to $V=V_{\text {eff }}, \tau=\left.\sigma\right|_{V_{\text {eff }}}$ and $V_{1}=\mathcal{T}_{(0, \mathbf{1}, 0)} Q \cap V_{\text {eff }}$ ) and conclude that

$$
\left.\sigma\right|_{V_{\text {eff }}} \text { is anti-symplectic. }
$$

For the scalar product $\langle\cdot, \cdot\rangle$ on $V_{\text {eff }}$ this means that we may assume in addition that it is invariant under $\left.\sigma\right|_{V_{\text {eff }}}$.

Write $V_{\text {eff }}=V_{\text {eff, } 1} \oplus V_{\text {eff,-1 }}$ for the decomposition of $V$ into eigenspaces of $\left.d \sigma\right|_{V_{\text {eff }}}$. Thus

$$
\left.d \sigma\right|_{V_{\text {eff }}}=\left(\begin{array}{cc}
\mathrm{id}_{V_{\text {eff, }}} & 0 \\
0 & -\mathrm{id}_{V_{\text {eff },-1}}
\end{array}\right) .
$$

Notice that $V_{\text {eff, } 1}=\mathcal{T}_{(0, \mathbf{1}, 0)} Q \cap V_{\text {eff. }}$.

Next we turn our attention to $\left.\sigma\right|_{W}$. From (2.1.5) and the concrete formula (2.2.2) for the $S_{m}$-action it follows that

$$
\left.\sigma\right|_{W}=\left(\begin{array}{ccc}
* & 0 & * \\
* & -\mathrm{id}_{\mathfrak{s}_{m}} & * \\
* & 0 & *
\end{array}\right)
$$

with respect to a basis of $W$ compatible with $W=\mathfrak{s}_{m}^{*} \times \mathfrak{s}_{m} \times V_{\text {fix }}$. A consequence of $(2.1 .3)$ is $d \Phi(0, \mathbf{1}, 0) \circ \sigma=d \Phi(0, \mathbf{1}, 0)$. From (2.2.4) it hence follows that 
$\left.d \Phi(0, \mathbf{1}, 0)\right|_{W} \circ \sigma_{W}=\left.d \Phi(0, \mathbf{1}, 0)\right|_{W}$. As $\left.d \Phi(0, \mathbf{1}, 0)\right|_{W}$ is the projection $W \rightarrow \mathfrak{s}_{m}^{*}$ along $\mathfrak{s}_{m} \times V_{\text {fix }}$, the pattern (2.2.8) further simplifies to

$$
\left.\sigma\right|_{W}=\left(\begin{array}{ccc}
\mathrm{id}_{\mathfrak{s}_{m}^{*}} & 0 & 0 \\
* & -\mathrm{id}_{\mathfrak{s}_{m}} & * \\
* & 0 & D
\end{array}\right)
$$

for some linear operator $D: V_{\text {fix }} \rightarrow V_{\text {fix }}$. As $\left.\left.\sigma\right|_{W} \circ \sigma\right|_{W}=\mathrm{id}_{W}$ we derive from (2.2.9) that $D^{2}=\mathrm{id}_{V_{\text {fix }}}$. Accordingly we obtain an eigenspace decomposition $V_{\text {fix }}=$ $V_{\text {fix }, 1} \oplus V_{\text {fix },-1}$ for $D$. With respect to the refined decomposition $W=\mathfrak{s}_{m}^{*} \times \mathfrak{s}_{m} \times$ $V_{\text {fix }, 1} \times V_{\text {fix },-1}$, we then have

$$
\left.\sigma\right|_{W}=\left(\begin{array}{cccc}
\mathrm{id}_{\mathfrak{s}_{m}^{*}} & 0 & 0 & 0 \\
A & -\mathrm{id}_{\mathfrak{s}_{m}} & B_{1} & B_{2} \\
C_{1} & 0 & \mathrm{id}_{V_{\mathrm{fix}, 1}} & 0 \\
C_{2} & 0 & 0 & -\mathrm{id}_{V_{\mathrm{fix},-1}}
\end{array}\right) .
$$

Employing again $\left.\left.\sigma\right|_{W} \circ \sigma\right|_{W}=\mathrm{id}_{W}$ we obtain that $B_{2}=0$ and $C_{1}=0$. Thus

$$
\left.\sigma\right|_{W}=\left(\begin{array}{cccc}
\mathrm{id}_{\mathfrak{s}_{m}^{*}} & 0 & 0 & 0 \\
A & -\mathrm{id}_{\mathfrak{s}_{m}} & B & 0 \\
0 & 0 & \mathrm{id}_{V_{\text {fix }, 1}} & 0 \\
C & 0 & 0 & -\mathrm{id}_{V_{\text {fix },-1}}
\end{array}\right)
$$

for linear operators $A: \mathfrak{s}_{\mathfrak{m}}^{*} \rightarrow \mathfrak{s}_{m}, B: V_{\text {fix }, 1} \rightarrow \mathfrak{s}_{m}$ and $C: \mathfrak{s}_{m}^{*} \rightarrow V_{\text {fix },-1}$. Combining (2.2.4) and (2.2.10) we then obtain

$$
\sigma=\left(\begin{array}{cccccc}
\mathrm{id}_{\mathfrak{s}_{m}^{*}} & 0 & 0 & 0 & & \\
A & -\mathrm{id}_{\mathfrak{s}_{m}} & B & 0 & & \\
0 & 0 & \mathrm{id}_{V_{\mathrm{fix}, 1}} & 0 & & \\
C & 0 & 0 & -\mathrm{id}_{V_{\text {fix },-1}} & \operatorname{id}_{V_{\text {eff }, 1}} & 0 \\
& & & & 0 & -\mathrm{id}_{V_{\text {eff },-1}}
\end{array}\right)
$$

with respect to a basis of $E$ compatible with $E=\mathfrak{s}_{m}^{*} \times \mathfrak{s}_{m} \times V_{\text {fix }, 1} \times V_{\text {fix },-1} \times V_{\text {eff, }, 1} \times$ $V_{\text {eff, }-1}$.

With the help of (2.2.11) we can now determine the tangent space $\mathcal{T}_{(0, \mathbf{1}, 0)} Q \subseteq E$. Notice that

$$
\mathcal{T}_{(0, \mathbf{1}, 0)} Q=\{v \in E: \sigma(v)=v\}
$$

so that (2.2.11) implies that

$$
\mathcal{T}_{(0, \mathbf{1}, 0)} Q=\left\{\left(\begin{array}{c}
x \\
\frac{1}{2}(A x+B y) \\
y \\
\frac{1}{2} C x \\
z \\
0
\end{array}\right): x \in \mathfrak{s}_{m}^{*}, y \in V_{\mathrm{fix}, 1}, z \in V_{\mathrm{eff}, 1}\right\} .
$$

Write pr: $E \rightarrow \mathfrak{s}_{m}^{*} \times V_{\text {fix }, 1} \times V_{\text {eff, } 1}$ for the projection along $\mathfrak{s}_{m} \times V_{\text {fix },-1} \times V_{\text {eff, }-1}$. Then (2.2.12) implies that $\mathbf{p r} \mid \mathcal{T}_{(0, \mathbf{1}, 0)} Q: \mathcal{T}_{(0, \mathbf{1}, 0)} \rightarrow \mathfrak{s}_{m}^{*} \times V_{\text {fix }, 1} \times V_{\text {eff, } 1}$ is a linear isomorphism. This in turn allows us to apply the implicit function theorem: there exist an open neigborhood $U_{1}$ of 0 in $\mathfrak{s}_{m}^{*} \times V_{\text {fix }, 1} \times V_{\text {eff, } 1}$, an open neigborhood $U_{2}$ of $(\mathbf{1}, 0,0)$ in $S_{m} \times V_{\text {fix },-1} \times V_{\text {eff,-1 }}$ and a differentiable map

$$
\psi=\left(\psi_{S}, \psi_{\mathrm{fix}}, \psi_{\mathrm{eff}}\right): U_{1} \rightarrow U_{2}
$$


such that $\psi(0,0,0)=(\mathbf{1}, 0,0)$ and

$$
Q \cap\left(U_{1} \times U_{2}\right)=\left\{\left(\begin{array}{c}
x \\
\psi_{S}(x, y, z) \\
y \\
\psi_{\text {fix }}(x, y, z) \\
z \\
\psi_{\text {eff }}(x, y, z)
\end{array}\right) \in \mathcal{T}^{*} S_{m} \times V:(x, y, z) \in U_{1}\right\} .
$$

One can say a little bit more about the map $\psi$ when we notice that $\mathcal{T}_{(0, \mathbf{1}, 0)} Q$ can equally be expressed as

$$
\mathcal{T}_{(0,1,0)} Q=\left\{\left(\begin{array}{c}
u \\
d \psi_{S}(0)(u, v, w) \\
v \\
d \psi_{\mathrm{fix}}(0)(u, v, w) \\
w \\
d \psi_{\mathrm{eff}}(0)(u, v, w)
\end{array}\right) \in E: u \in \mathfrak{s}_{m}^{*}, v \in V_{\mathrm{fix}, 1}, w \in V_{\mathrm{eff}, 1}\right\}
$$

Comparing (2.2.12) with (2.2.14) yields

$$
d \psi_{\text {eff }}(0)=0 .
$$

We are now ready to summarize the discussion of this subsection. In more compact notation we have proved the following:

Theorem 2.2.2 (Local normal forms for $\Phi$ and $\left.\Phi\right|_{Q}$ ). Let $(M, \omega)$ be a connected symplectic manifold endowed with a Hamiltonian torus action and an involution $\tau: M \rightarrow M$ which satisfy (2.1.2)-(2.1.4). Let $m \in Q$. Write $k=\operatorname{dim} \mathfrak{s}_{m}=\operatorname{dim} \mathfrak{t} / \mathfrak{t}_{m}$ and identify $\mathfrak{t}^{*} \simeq \mathbb{R}^{k} \times \mathfrak{t}_{m}^{*}$. Then there exist an open neighborhood $U$ of $m$ and symplectic coordinates $\mathbf{x}, \mathbf{y}, \mathbf{q}, \mathbf{p}=x_{1}, \ldots, x_{k}, y_{1}, \ldots, y_{k}, q_{1}, \ldots, q_{N}, p_{1}, \ldots, p_{N}$ with $\mathbf{x}(m)=\mathbf{y}(m)=\mathbf{q}(m)=\mathbf{p}(m)=0$ such that:

1. On $U$ the momentum map $\Phi: M \rightarrow \mathfrak{t}^{*} \simeq \mathbb{R}^{k} \times \mathfrak{t}_{m}^{*}$ is given by

$$
\Phi=\Phi(m)+\left(\mathbf{x}, \frac{1}{2} \sum_{j=1}^{l} \lambda_{j}\left(q_{j}^{2}+p_{j}^{2}\right)\right)
$$

where $l \leq N$ and $\lambda_{1}, \ldots, \lambda_{l} \in \mathfrak{t}^{*} \backslash\{0\}$.

2. The restriction $(\mathbf{x}, \mathbf{q}): Q \cap U \rightarrow \mathbb{R}^{n}$ of $(\mathbf{x}, \mathbf{q})$ to $Q \cap U$ is a diffeomorphism onto an open ball $B_{r}^{n}(0)$ of radius $r>0$ in $\mathbb{R}^{n}$. Furthermore

$$
\left.\Phi\right|_{Q}=\Phi(m)+\left(\mathbf{x}, \frac{1}{2} \sum_{j=1}^{l} \lambda_{j}\left(q_{j}^{2}+\psi_{j}(\mathbf{x}, \mathbf{q})^{2}\right)\right),
$$

where $\psi=\left(\psi_{1}, \ldots, \psi_{l}\right): B_{r}^{n}(0) \rightarrow \mathbb{R}^{l}$ is a differentiable map with $\psi(0)=0$ and $d \psi(0)=0$.

Proof. To explain the notation in the theorem: $(\mathbf{x}, \mathbf{y})$ are symplectic coordinates for $\mathcal{T}^{*} S_{m}=\mathfrak{s}_{m}^{*} \times S_{m}$ with $\mathbf{x}$ corresponding to $\mathfrak{s}_{m}^{*}$ and $\mathbf{y}$ to $S_{m} ;$ next $(\mathbf{q}, \mathbf{p})$ are symplectic coordinates for $V$ compatible with the weight space decomposition $V=\bigoplus_{\lambda} V_{\lambda}$ and moreover $q_{1}, \ldots, q_{l}$ corresponding to $V_{\text {eff, } 1}$ and $q_{l+1}, \ldots, q_{N}$ corresponding to $V_{\text {fix }, 1}$ (and similarly for $\mathbf{p}$ ). 
The expression for $\Phi$ in 1. then follows from (2.2.3) and (2.2.6) which implied that the inner product on $V_{\text {eff }}$ could be chosen $\left.\sigma\right|_{V_{\text {eff }}}$-invariant. Finally, the assertion in 2. follows from 1. and (2.2.13) combined with (2.2.15). Here the map $\psi$ corresponds to $\psi_{\text {eff }}$ above.

\section{The CONVEXITY THEOREM}

The objective of this subsection is to prove

Theorem 3.1. Let $M$ be a compact connected symplectic manifold with Hamiltonian torus action $T \times M \rightarrow M$ and momentum map $\Phi: M \rightarrow \mathfrak{t}^{*}$. Further let $\tau: M \rightarrow M$ be an involutive diffeomorphism with fixed point set $Q$ such that

1. $t \circ \tau=\tau \circ t^{-1}$ for all $t \in T$.

2. $\Phi \circ \tau=\Phi$.

3. $Q$ is a Lagrangian submanifold of $M$.

Then $\Phi(Q)=\Phi(M)$. In particular $\Phi(Q)$ is a convex subset of $\mathfrak{t}^{*}$. Moreover, the same assertions hold if $Q$ is replaced with any of its connected components.

Our arguments follow the approach of Duistermaat (cf. 4, Sect. 2]). However, they have to be adapted to the more general case where $\tau$ is not necessarily antisymplectic.

From now on we will assume that $M$ is compact. In particular, $Q$ is a compact submanifold of $M$. Furthermore, we will require that $T$ acts freely, i.e. $\bigcap_{m \in M} T_{m}=$ $\{\mathbf{1}\}$. But notice that this is not a severe restriction, as we can always replace $T$ with $T / \bigcap_{m \in M} T_{m}$.

The key result toward convexity of $\Phi(Q)$ is the following central fact which generalizes [6, Lemma 32.1] and [4, Lemma 2.4].

Proposition 3.2. Let $X \in \mathfrak{t}$. Then the function $\left.\Phi_{X}\right|_{Q}$ has a unique local maximal value.

Proof. Fix $X \in \mathfrak{t}$ and let $m \in Q$ be a critical point of $\left.\Phi_{X}\right|_{Q}$. By Lemma 2.2.1 we know that $m$ is fixed by $\exp (\mathbb{R} X)$. Thus replacing $T$ by $\overline{\exp (\mathbb{R} X)}$ we may assume that $d\left(\left.\Phi\right|_{Q}\right)(m)=0$. In particular we obtain from Theorem 2.2 .2 with $\mathfrak{s}_{m}=\{0\}$ that

$$
\Phi_{X}=\Phi_{X}(m)+\frac{1}{2} \sum_{j=1}^{l} \lambda_{j}(X)\left(q_{j}^{2}+p_{j}^{2}\right)
$$

and

$$
\left.\Phi_{X}\right|_{Q}=\Phi_{X}(m)+\frac{1}{2} \sum_{j=1}^{l} \lambda_{j}(X)\left(q_{j}^{2}+\psi_{j}(\mathbf{q})^{2}\right)
$$

hold in a neighborhood of $U$ of $m$.

Claim. $m$ is a local maximum for $\left.\Phi\right|_{Q}$ iff $\lambda_{j}(X) \leq 0$ for all $1 \leq j \leq l$.

In view of (3.1) this is clear if all $\lambda_{j}(X) \leq 0$. To obtain the other direction assume that $\lambda_{j}(X)>0$ for some $j$. W.l.o.g. we may assume that $\lambda_{1}(X)>0$. For each $j$ we then have

$$
\psi_{j}\left(q_{1}, 0, \ldots, 0\right)=q_{1}^{2} h_{j}\left(q_{1}\right)
$$


for a continuous function $h_{j}$. This is because of $\psi_{j}(0)=d \psi_{j}(0)=0$ for all $1 \leq j \leq l$ (see Theorem 2.2.2 2.). Thus for small and non-zero $q_{1}$ we obtain from (3.2)

$$
\begin{aligned}
\left.\Phi_{X}\right|_{Q}\left(q_{1}, 0, \ldots, 0\right) & =\Phi_{X}(m)+\frac{1}{2}\left(\lambda_{1}(X) q_{1}^{2}\left(1+q_{1}^{2} h_{1}\left(q_{1}\right)^{2}\right)\right. \\
& \left.+q_{1}^{4} \sum_{j=2}^{l} \lambda_{j}(X) h_{j}\left(q_{1}\right)^{2}\right) \leq \Phi_{X}(m) .
\end{aligned}
$$

Dividing by $\frac{1}{2} q_{1}^{2}$ implies that

$$
\lambda_{1}(X)\left(1+q_{1}^{2} h_{1}\left(q_{1}\right)^{2}\right)+q_{1}^{2} \sum_{j=2}^{l} \lambda_{j}(X) h_{j}\left(q_{1}\right)^{2} \leq 0
$$

for $q_{1}$ small and non-zero. This clearly contradicts $\lambda_{1}(X)>0$ and proves our claim.

It follows from our claim and (3.1) and (3.2) that $m$ is a local maximum for $\left.\Phi_{X}\right|_{Q}$ if and only if $m$ is a local maximum for $\Phi_{X}$. As $M$ is compact, Morse theoretic arguments imply that the set of points of $M$, where $\Phi_{X}$ attains a local maximum, is connected (see the proof of [6, Cor. 32.1] or [4, Lemma 2.4]). This completes the proof of the proposition.

Lemma 3.3. If $m \in Q$ is such that $\xi=\Phi(m)$ is a boundary point of $\Phi(Q)$, then $\mathfrak{t}_{m} \neq\{0\}$.

Proof. If $\mathfrak{t}_{m}=\{0\}$, then (2.2.1) implies that $d\left(\left.\Phi\right|_{Q}\right)(m)$ is surjective, contradicting the fact that $\xi$ is a boundary point.

Let $\left.\mathbb{R}^{+}=\right] 0, \infty\left[\right.$ and $\mathbb{R}_{0}^{+}=\left[0, \infty\left[\right.\right.$ and define a closed convex cone in $\mathfrak{t}_{m}^{*}$ by

$$
\Gamma_{m}=\left\{\sum_{j=1}^{l} s_{j} \lambda_{j}: s=\left(s_{1}, \ldots, s_{l}\right) \in\left(\mathbb{R}_{0}^{+}\right)^{l}\right\} .
$$

Lemma 3.4. If $\Gamma_{m}=\mathfrak{t}_{m}^{*}$, then $\left.\operatorname{im} \Phi\right|_{Q}$ contains an open neighborhood of $\Phi(m)$ in $\mathfrak{t}_{m}^{*}$.

Proof. Since $\Gamma_{m}=\mathfrak{t}_{m}^{*}$, there exists a vector

$$
\mathbf{v}=\left(0 ; v_{1}, \ldots, v_{l}\right) \in \mathbb{R}^{k} \times\left(\mathbb{R}^{+}\right)^{l} \quad \text { such that } \sum_{j=1}^{n} v_{j} \lambda_{j}=0 \in \mathfrak{t}_{m}^{*} .
$$

Then for any neighborhood $U$ of $\mathbf{v} \in \mathbb{R}^{k} \times\left(\mathbb{R}^{+}\right)^{l}$ the set

$$
\left\{\left(u_{1}, \ldots, u_{k}, u_{k+1} \lambda_{1}+\cdots+u_{n} \lambda_{l}\right):\left(u_{1}, \ldots, u_{n}\right) \in U\right\}
$$

contains an open neighborhood of 0 in $t^{*}$.

Recall the map $\psi: B_{r}^{n}(0) \rightarrow \mathbb{R}^{l}$ from Theorem 2.2.2 2. We define

$$
\Psi: B_{r}^{n}(0) \rightarrow \mathbb{R}^{k} \times\left(\mathbb{R}_{0}^{+}\right)^{l}, \quad(\mathbf{x}, \mathbf{q}) \mapsto\left(\mathbf{x}, \frac{1}{2}\left(q_{1}^{2}+\psi_{1}(\mathbf{x}, \mathbf{q})^{2}\right), \ldots, \frac{1}{2}\left(q_{l}^{2}+\psi_{l}(\mathbf{x}, \mathbf{q})^{2}\right)\right) .
$$

According to Theorem 2.2.2 2. it is sufficient to show that im $\Psi$ contains a point $\mathbf{v}$ as in (3.3) as an inner point. Fix $\mathbf{v}$ satisfying (3.3). The theorem will be proved if we can show that $\operatorname{im} \Psi$ contains $s \mathbf{v}$ as an inner point for some $s>0$. In the following we will verify this claim. We may assume that $\|\mathbf{v}\|=1$. 
The properties $\psi(0)=0, d \psi(0)=0$ from Theorem 2.2.2 2. are crucial. Together with Taylor's formula they imply that we can find a constant $K>0$ such that for all $1 \leq j \leq l$,

$$
\left|\psi_{j}(\mathbf{x}, \mathbf{q})\right| \leq K\|(\mathbf{x}, \mathbf{q})\|^{2},
$$

for every $(\mathbf{x}, \mathbf{q}) \in \mathbb{R}^{k} \times\left(\mathbb{R}^{+}\right)^{l}$ sufficiently close to $(0,0)$.

There is another constant $C>0$ such that the ball $B_{s C}^{n}(s \mathbf{v})$ lies entirely in $\mathbb{R}^{k} \times\left(\mathbb{R}^{+}\right)^{l}$ for all $s>0$. We notice that every point in $B_{s C}^{n}(s \mathbf{v})$ can be written as $\left(x_{1}, \ldots, x_{k}, \frac{1}{2} q_{1}^{2}, \ldots, w \frac{1}{2} q_{l}^{2}\right)$ with unique $x_{1}, \ldots, x_{k} \in \mathbb{R}, q_{1}, \ldots, q_{l} \in \mathbb{R}^{+}$. From now on let $0<s \leq 1$. Observe that the condition

$$
\left(x_{1}, \ldots, x_{k}, \frac{1}{2} q_{1}^{2}, \ldots, \frac{1}{2} q_{l}^{2}\right) \in B_{s C}^{n}(s \mathbf{v})
$$

puts restrictions on the vector $\left(x_{1}, \ldots, x_{k}, q_{1}, \ldots, q_{l}\right)$ : there is a constant $\widetilde{C}>0$ (independent of the vector) such that

$$
\left\|\left(x_{1}, \ldots, x_{k}, q_{1}, \ldots, q_{l}\right)\right\| \leq \sqrt{s} \widetilde{C} .
$$

In particular, (3.5) implies that for some $\widetilde{K}>0$,

$$
\|\left(0, \ldots, 0, \frac{1}{2} \psi_{1}(\mathbf{x}, \mathbf{q})^{2}, \ldots, \frac{1}{2} \psi_{l}(\mathbf{x}, \mathbf{q})^{2} \| \leq \widetilde{K} s^{2}\right.
$$

for all $(\mathbf{x}, \mathbf{q})=\left(x_{1}, \ldots, x_{k}, q_{1}, \ldots, q_{l}\right)$ with $\left(x_{1}, \ldots, x_{k}, \frac{1}{2} q_{1}^{2}, \ldots, \frac{1}{2} q_{l}^{2}\right) \in B_{s C}^{n}(s \mathbf{v})$.

Choose $0<s_{0} \leq 1$ small enough such that $\widetilde{K} s_{0}^{2}<s_{0} C$ holds. Set $\epsilon=s_{0} C$. We are now in a position to apply Brouwer's fixed point theorem: consider the mapping

$$
\begin{gathered}
\Xi: \overline{B_{\epsilon}^{n}(0)} \rightarrow \overline{B_{\epsilon}^{n}(0)}, \\
\left(x_{1}, \ldots, x_{k}, \frac{1}{2} q_{1}^{2}, \ldots, \frac{1}{2} q_{l}^{2}\right)-s_{0} \mathbf{v} \mapsto-\left(0, \ldots, 0, \frac{1}{2} \psi_{1}(\mathbf{x}, \mathbf{q})^{2}, \ldots, \frac{1}{2} \psi_{l}(\mathbf{x}, \mathbf{q})^{2}\right) .
\end{gathered}
$$

If $\left(\mathbf{x}, \frac{1}{2} q_{1}^{2}, \ldots, \frac{1}{2} q_{l}^{2}\right)-s_{0} \mathbf{v}$ is a fixed point of $\Xi$, then

$$
\Psi(\mathbf{x}, \mathbf{q})=\left(\mathbf{x}, \frac{1}{2}\left(q_{1}^{2}+\psi_{1}(\mathbf{x}, \mathbf{q})^{2}\right), \ldots, \frac{1}{2}\left(q_{l}^{2}+\psi_{l}(\mathbf{x}, \mathbf{q})^{2}\right)\right)=s_{0} \mathbf{v} .
$$

We want to show that $s_{0} \mathbf{v}$ is an inner point of im $\Psi$. Notice that by choosing $s_{0}$ small enough we can assume that the point $(\mathbf{x}, \mathbf{q})$ with $\Psi(\mathbf{x}, \mathbf{q})=s_{0} \mathbf{v}$ is arbitrarily close to $(0,0)$. The mapping $\Psi$ is submersive at most points close enough to the origin as a look at its derivative shows:

$$
\begin{aligned}
d \Psi(\mathbf{x}, \mathbf{q}) & =\left(\begin{array}{cccc}
\operatorname{id}_{k} & & & \\
& q_{1} & & \\
& & \ddots & \\
& & & q_{l}
\end{array}\right) \\
& +\left(\begin{array}{ccc}
0 & 0 \\
\left(\psi_{i}(\mathbf{x}, \mathbf{q}) \frac{\partial \psi_{i}}{\partial x_{j}}(\mathbf{x}, \mathbf{q})\right)_{i, j} & \left(\psi_{i}(\mathbf{x}, \mathbf{q}) \frac{\partial \psi_{i}}{\partial q_{j}}(\mathbf{x}, \mathbf{q})\right)_{i, j}
\end{array}\right) .
\end{aligned}
$$

Relation (3.5) implies that for $(\mathbf{x}, \mathbf{q})$ approaching $(0,0)$ the entries in the second summand become arbitrarily small compared to those in the first summand. Since the $\mathbf{q}$ under consideration satisfy $q_{1}, \ldots, q_{l}>0$, we conclude that

$$
\operatorname{det}(\Psi(\mathbf{x}, \mathbf{q}))>0 \text { for }(\mathbf{x}, \mathbf{q}) \text { close enough to }(0,0) .
$$

This finishes the proof. 
If $C$ is a closed convex cone in an Euclidean vector space $E$, then its dual cone in $E^{*}$ is defined by

$$
C^{\star}=\left\{\lambda \in E^{*}:\left.\lambda\right|_{C} \geq 0\right\} .
$$

Recall that $C^{\star}$ is a closed convex cone in $E^{*}$. One has $\left(C^{\star}\right)^{\star}=C$, and in particular $C^{\star}=\{0\}$ if and only if $C=E$.

Lemma 3.5. Let $\xi \in \Phi(Q)$ be a boundary point and let $m \in Q$ be such that $\Phi(m)=\xi$. Then the following assertions hold:

1. $\Gamma_{m} \neq \mathfrak{t}_{m}^{*}$. In particular $\{0\} \neq \Gamma_{m}^{\star} \subseteq \mathfrak{t}_{m}$.

2. For all $0 \neq X \in-\Gamma_{m}^{\star} \subseteq \mathfrak{t}_{m}$, one has $\left.\Phi_{X}\right|_{Q} \leq \Phi_{X}(m)=\langle\xi, X\rangle$. In particular $\operatorname{im} \Phi$ is contained in the half space $\left\{\lambda \in \mathfrak{t}^{*}:(\xi-\lambda)(X) \geq 0\right\}$.

3. $\xi$ is a boundary point of $\Phi(M)$.

Proof. 1. Suppose that $\Gamma_{m}=\mathfrak{t}_{m}^{*}$. Then Lemma 3.4 implies that im $\Phi$ contains a neighborhood of $\xi$. But this contradicts the fact that $\xi$ is a boundary point.

2. According to 1 . we have $\Gamma_{m}^{\star} \neq\{0\}$. Let $0 \neq X \in-\Gamma_{m}^{\star}$. Then (3.2) implies that $\Phi_{X}\left(m^{\prime}\right) \leq \Phi_{X}(m)=\xi(X)$ for all $m^{\prime}$ in a small neighborhood of $m$ in $Q$. Thus $\Phi_{X}(m)$ is a local maximal value of $\left.\Phi_{X}\right|_{Q}$. Hence Proposition 3.2 implies that $\left.\Phi_{X}\right|_{Q} \leq \Phi_{X}(m)$. This completes the proof of 2 .

3. A slight modification (refer to (3.1) instead of (3.2)) of the argument just given shows that there is a $0 \neq X \in-\Gamma_{m}^{\star}$ such that

$$
\Phi_{X}\left(m^{\prime}\right) \leq \Phi_{X}(m)=\langle\xi, X\rangle \quad \text { for all } m^{\prime} \text { in a neighborhood of } m \text { in } M .
$$

The function $\Phi_{X}$ on $M$ has a unique local maximal value (see [4, Lemma 2.4]). Therefore, $\Phi(M)$ must lie entirely in the halfspace $\left\{\lambda \in \mathfrak{t}^{*}:(\xi-\lambda)(X) \geq 0\right\}$, implying that $\xi$ is a boundary point of $\Phi(M)$.

Let us define regular elements in $Q$ by

$$
Q_{\text {reg }}=\left\{m \in Q:\left.d \Phi\right|_{Q}(m) \text { is surjective }\right\} .
$$

Lemma 3.6. The following assertions hold:

1. $Q_{\text {reg }}$ is open and dense in $Q$.

2. The interior $\operatorname{int} \Phi(Q)$ is dense in $\Phi(Q)$.

Proof. 1. It is clear that $Q_{\text {reg }}$ is open. Let us show that $Q_{\text {reg }}$ is dense. For that fix $m \in Q \backslash Q_{\text {reg. }}$. Then $\left.d \Phi\right|_{Q}(m)$ is not onto and hence $\mathfrak{t}_{m} \neq\{0\}$ by (2.2.1). In the $(x, \mathbf{q})$-coordinates near $m$ we have

$$
\Phi_{Q}(\mathbf{x}, \mathbf{q})=\Phi(m)+\left(\mathbf{x}, \frac{1}{2} \sum_{j=1}^{l}\left(q_{j}^{2}+\psi_{j}(\mathbf{x}, \mathbf{q})^{2}\right) \lambda_{j}\right)
$$

(cf. Theorem 2.2.2 2.). As we assume that $\bigcap_{m \in M} T_{m}=\{\mathbf{1}\}$, it follows that $\lambda_{1}, \ldots, \lambda_{l}$ linearly span $\mathfrak{t}_{m}^{*}$. Recall the definition of the map $\Psi$ from (3.4). As was shown at the end of the proof of Lemma 3.4 we can find elements $(0, \mathbf{q})$ arbitrarily close to $m=(0,0)$ such that $\left.d \Psi\right|_{Q}(0, \mathbf{q})$ is invertible. This in turns implies that $\left.d \Phi\right|_{Q}(0, \mathbf{q})$ is surjective and completes the proof of 1 .

2. This is immediate from 1 .

Proof of Theorem 3.1. We first show that $\Phi(Q)$ is convex. For that notice that $\Phi(Q)$ is a compact subset of $\boldsymbol{t}^{*}$ with open and dense interior (Lemma 3.62 .). By 12, Satz 3.3] $\Phi(Q)$ will be convex if each boundary point lies on a half space containing $\Phi(Q)$. In view of Lemma 3.5 2. this is satisfied and so $\Phi(Q)$ is convex. 
Next we show that $\Phi(Q)=\Phi(M)$. Assume that some extremal point $\eta$ of $\Phi(M)$ does not lie in $\Phi(Q)$. From Lemma 3.6 we know there is a point $\zeta$ in int $\Phi(Q)$. On the line segment connecting $\eta$ and $\zeta$ there must be a boundary point $\beta$ of $\Phi(Q)$. Since $\Phi(M)$ is a convex polyhedron (and since $\eta \notin \Phi(Q)$ ), we see that $\beta$ must be contained in int $\Phi(M)$. This contradicts Lemma 3.53 .

\section{Application to Kostant's theorem}

In this section we will use Theorem 3.1 to give a symplectic proof of Kostant's non-linear convexity theorem. We start with the introduction of the necessary notation and the statement of Kostant's result.

4.1 Notation. Let $G$ denote a connected semisimple Lie group. Universal complexifications of Lie groups will be denoted by a subscript $\mathbb{C}$, i.e. $G_{\mathbb{C}}$ is the universal complexification of $G$, etc. For what follows it is no loss of generality when we assume that $G \subseteq G_{\mathbb{C}}$ and that $G_{\mathbb{C}}$ is simply connected.

Write $\mathfrak{g}$ for the Lie algebra of $G$. Complexifications of Lie algebras shall be denoted by the subsript $\mathbb{C}$, i.e. $\mathfrak{g}_{\mathbb{C}}$ is the complexification of $\mathfrak{g}$, etc.

Let $\mathfrak{g}=\mathfrak{k}+\mathfrak{p}$ be a Cartan decomposition of $\mathfrak{g}$ with $\mathfrak{k}$ a maximal compact subalgebra. Fix a maximal abelian subspace $\mathfrak{a} \subseteq \mathfrak{p}$ and denote by $\Sigma=\Sigma(\mathfrak{g}, \mathfrak{a})$ the corresponding restricted root system in $\mathfrak{a}^{*}$, the dual of $\mathfrak{a}$. For each $\alpha \in \Sigma$ let $\mathfrak{g}^{\alpha}=\{Y \in \mathfrak{g}:(\forall H \in \mathfrak{a})[H, Y]=\alpha(H)\}$ be the associated root space. With $\mathfrak{m}=\mathfrak{z k}_{\mathfrak{k}}(\mathfrak{a})$ one then has the root space decomposition

$$
\mathfrak{g}=\mathfrak{a}+\mathfrak{m}+\bigoplus_{\alpha \in \Sigma} \mathfrak{g}^{\alpha}
$$

Select a positive system $\Sigma^{+} \subseteq \Sigma$ and define the nilpotent subalgebra $\mathfrak{n}=\bigoplus_{\alpha \in \Sigma^{+}} \mathfrak{g}^{\alpha}$.

On the group level we denote by $A, K$ and $N$ the analytic subgroups of $G$ with Lie algebras $\mathfrak{a}, \mathfrak{k}$ and $\mathfrak{n}$. Then there is the Iwasawa decomposition of $G$ which states that the multiplication mapping

$$
N \times A \times K \rightarrow G, \quad(n, a, k) \mapsto n a k
$$

is an analytic diffeomorphism. For $g \in G$ let us denote by $\widetilde{a}(g)$ the $A$-component of $g$ in the Iwasawa decomposition.

Set $M=Z_{K}(\mathfrak{a})$ and note that the Lie algebra of $M$ is $\mathfrak{m}$. The Weyl group of $\Sigma$ can then be defined by $\mathcal{W}=N_{K}(\mathfrak{a}) / M$.

We are ready to state Kostant's theorem [11:

Theorem 4.1.1. Let $Y \in \mathfrak{a}$. Then

$$
\log \widetilde{a}(K \exp (Y))=\operatorname{conv}(\mathcal{W} \cdot Y),
$$

where $\operatorname{conv}(\cdot)$ denotes the convex hull of $(\cdot)$.

4.2 Symplectic methods for the complex case. In case $G$ was complex, a symplectic proof of Theorem 4.1.1 was given by Lu and Ratiu [13. The objective of this section is to briefly recall their method.

Let us assume that $G$ is complex, i.e. $\mathfrak{g}$ is a complex Lie algebra. Then the Cartan decomposition of $\mathfrak{g}$ is given by $\mathfrak{g}=\mathfrak{k}+i \mathfrak{k}$, i.e. $\mathfrak{p}=i \mathfrak{k}$. Furthermore $\mathfrak{a}=i \mathfrak{t}$ with $\mathfrak{t}$ a maximal toral subalgebra in $\mathfrak{k}$. Set $T=\exp (\mathfrak{t})$.

Define a solvable subalgebra of $\mathfrak{g}$ by $\mathfrak{b}=\mathfrak{a}+\mathfrak{n}$ (despite the notation that this is not a Borel subalgebra of $\mathfrak{g}$ ). Write $B=A N$ for the corresponding group and notice that $B$ is invariant under conjugation by the torus $T$. 
Let us denote by $\kappa$ the Cartan-Killing form of the complex Lie algebra $\mathfrak{g}$ and define a symmetric $\mathbb{R}$-valued bilinear form on $\mathfrak{g}$ by

$$
\mathrm{B}: \mathfrak{g} \times \mathfrak{g} \rightarrow \mathbb{R}, \quad \mathrm{B}(X, Y)=\Im \kappa(X, Y) .
$$

Notice that $\mathrm{B}$ is invariant and non-degenerate. The important fact is that both $\mathfrak{b}$ and $\mathfrak{k}$ are isotropic for $\mathrm{B}$; in other words $(\mathfrak{g}, \mathfrak{b}, \mathfrak{k})$ becomes a Manin-triple 3 . Likewise $(G, B, K)$ is a Manin-triple. Recall that this implies that $B \simeq G / K$ carries a natural structure of a Poisson Lie group [3. In order to describe the symplectic leaves write $\widetilde{b}(g)$ for the $B$-part of $g \in G$ in the decomposition $G=B \cdot K$. Then the symplectic leaves in $B$ are given by $M_{a}=\widetilde{b}(K a)$ for $a \in A$. As manifolds, $M_{a} \simeq K / K_{a}$ with $K_{a}=Z_{K}(a)$. Notice that $K$ does not act symplectically on $M_{a}$; however $T$ does, and the $T$-action is Hamiltonian. It was established in 13 that the corresponding momentum map is the non-linear Iwasawa projection:

$$
\Phi: M_{a} \rightarrow \mathfrak{a} \simeq \mathfrak{t}^{*}, \quad \widetilde{b}(k a) \mapsto \log \widetilde{a}(k a) .
$$

Standard structure theory implies that $\operatorname{Fix}\left(M_{a}\right)=\mathcal{W} . a$. Thus (4.2.1) combined with the Atiyah-Guillemin-Sternberg convexity theorem gives a symplectic proof of Theorem 4.1.1 in the case of $G$ complex [13].

For later reference we give an explicit formula for the symplectic form on a leaf $M_{a}=\widetilde{b}(K a)$. For $X \in \mathfrak{k}$ let us denote by $\widetilde{X}$ the corresponding vector field on $M_{a}$, i.e.

$$
\widetilde{X}_{b}=\left.\frac{d}{d t}\right|_{t=0} \widetilde{b}(\exp (t X) b) \quad\left(b \in M_{a}\right) .
$$

Then the symplectic form $\omega$ of $M_{a}$ is given by

$$
\omega_{b}\left(\widetilde{X}_{b}, \widetilde{Y}_{b}\right)=\mathrm{B}\left(\mathbf{p r}_{\mathfrak{k}}\left(\operatorname{Ad}(b)^{-1} X\right), \operatorname{Ad}(b)^{-1} Y\right) \quad\left(b \in M_{a} ; X, Y \in \mathfrak{k}\right),
$$

where $\mathbf{p r}_{\mathfrak{k}}: \mathfrak{g} \rightarrow \mathfrak{k}$ is the projection along $\mathfrak{b}$. This is immediate from [3, (11.1.2)].

4.3 Symplectic methods for the real case. Define a maximal compact subalgebra $\mathfrak{u}$ in $\mathfrak{g}_{\mathbb{C}}$ by $\mathfrak{u}=\mathfrak{k}+i \mathfrak{p}$ and let $U$ be the corresponding maximal compact subgroup of $G_{\mathbb{C}}$. Notice that $\mathfrak{g}_{\mathbb{C}}=\mathfrak{u}+i \mathfrak{u}$ is a Cartan decomposition of $\mathfrak{g}_{\mathbb{C}}$. If $\mathfrak{t}_{1}$ denotes a maximal torus in $\mathfrak{m}=\mathfrak{z}_{\mathfrak{k}}(\mathfrak{a})$, then $\mathfrak{a}_{1}=\mathfrak{a}+i \mathfrak{t}_{1}$ defines a maximal abelian subspace of $i \mathfrak{u}$. Write $\Sigma_{1}=\Sigma_{1}\left(\mathfrak{g}_{\mathbb{C}}, \mathfrak{a}_{1}\right)$ for the corresponding root system. Fix a positive system $\Sigma_{1}^{+}$of $\Sigma_{1}$. Without loss of generality we may assume that $\Sigma_{1}^{+}$and $\Sigma^{+}$are compatible, i.e. $\left.\Sigma_{1}^{+}\right|_{\mathfrak{a}} \subseteq \Sigma^{+} \cup\{0\}$. Write $\mathfrak{n}_{1}=\bigoplus_{\alpha \in \Sigma_{1}^{+}} \mathfrak{g}_{\mathbb{C}}^{\alpha}$ for the nilpotent subalgebra of $\mathfrak{g}_{\mathbb{C}}$ associated to $\Sigma_{1}^{+}$. Likewise we denote by $N_{1}$ the corresponding subgroup of $G_{\mathbb{C}}$. Notice that $N_{\mathbb{C}} \subseteq N_{1}$ but generally $N_{\mathbb{C}} \subsetneq N_{1}$ unless $\mathfrak{m}=\mathfrak{t}_{1}$ is abelian. Clearly we have $A \subseteq A_{1}$ and so $B \subseteq B_{1}$, where $B=A N$ and $B_{1}=A_{1} N_{1}$. Finally let us define the torus $T=\exp (i \mathfrak{a})$.

Fix $a \in A \subseteq A_{1}$ and consider the symplectic manifold $M_{a}=\widetilde{b}(U a)$ of the Poisson Lie group $B_{1}$ (cf. Subsection 4.2). As explained in Subsection 4.2 the action of $T$ on $M_{a}$ is Hamiltonian with momentum map $\Phi: M_{a} \rightarrow \mathfrak{a} \simeq \mathfrak{t}^{*}$ given by (4.2.2)

$$
\Phi\left(\widetilde{b}_{1}(u a)\right)=\log \widetilde{a}(u g) \quad(u \in U)
$$

with $\widetilde{a}(g)$ the $A$-part of $g \in G_{\mathbb{C}}$ in the decomposition $G_{\mathbb{C}}=N_{1} A \exp \left(i \mathfrak{t}_{1}\right) U$.

Set $Q_{a}=\widetilde{b}(K a)$. Then the restriction of $\Phi$ to $Q_{a}$ is the non-linear Iwasawa projection, i.e $\Phi(\widetilde{b}(k a))=\log \widetilde{a}(k a)$ for all $k \in K$. We are interested in the image $\Phi\left(Q_{a}\right)$. 
Denote by $\tau: G_{\mathbb{C}} \rightarrow G_{\mathbb{C}}$ the complex conjugation with respect to the real form $G$. Notice that $\tau$ is an involutive diffeomorphism of $G_{\mathbb{C}}$ which induces an involution on $M_{a}$, say $\tau_{a}$, by the prescription

$$
\tau_{a}(\widetilde{b}(u a))=\widetilde{b}(\tau(u a))=\widetilde{b}(\tau(u) a) \quad(u \in U) .
$$

Standard stucture theory shows that the connected component containing $a \in M_{a}$ of the fixed-point set of the involution $\tau_{a}: M_{a} \rightarrow M_{a}$ is given by $Q_{a}=\widetilde{b}(K a)$. We collect some important properties of $\tau_{a}$ and $Q_{a}$ :

Lemma 4.3.1. The following assertions hold:

1. $Q_{a} \subseteq M_{a}$ is a Lagrangian submanifold of $M_{a}$.

2. For all $t \in T$ and $m \in M_{a}$ one has $\tau_{a}(t . m)=t^{-1} \cdot \tau_{a}(m)$.

3. $\Phi \circ \tau_{a}=\Phi$.

Proof. 1. It is sufficient to show that $\omega_{b}\left(\widetilde{X}_{b}, \widetilde{Y}_{b}\right)=0$ for all $b \in Q_{a}$ and $X, Y \in \mathfrak{k}$. Notice that $B$ and $\mathfrak{k}$ are $\tau$-fixed. Thus (4.2.2) implies that

$$
\begin{aligned}
\omega_{b}\left(\tilde{X}_{b}, \tilde{Y}_{b}\right) & =\mathrm{B}\left(\mathbf{p r}_{\mathfrak{u}}\left(\operatorname{Ad}(b)^{-1} X\right), \operatorname{Ad}(b)^{-1} Y\right) \\
& =\mathrm{B}\left(\mathbf{p r}_{\mathfrak{k}}\left(\tau\left(\operatorname{Ad}(b)^{-1} X\right)\right), \tau\left(\operatorname{Ad}(b)^{-1} Y\right)\right) \\
& =\mathrm{B}\left(\tau\left(\mathbf{p r}_{\mathfrak{k}}\left(\operatorname{Ad}(b)^{-1} X\right)\right), \tau\left(\operatorname{Ad}(b)^{-1} Y\right)\right) .
\end{aligned}
$$

By the definition of $\mathrm{B}$ we have $\mathrm{B} \circ(\tau \times \tau)=-\mathrm{B}$. Thus our computation above gives $\omega_{b}\left(\widetilde{X}_{b}, \widetilde{Y}_{b}\right)=-\omega_{b}\left(\widetilde{X}_{b}, \widetilde{Y}_{b}\right)$; completing the proof of 1 .

2. Let $b \in M_{a}$. Then $b=\widetilde{b}(u a)$ for some $u \in U$. Hence for $t \in T$,

$$
\tau_{a}(t . b)=\tau_{a}(\widetilde{b}(t u a))=\widetilde{b}(\tau(t u a))=\widetilde{b}\left(t^{-1} \tau(u a)\right)=t^{-1} \cdot \tau_{a}(b),
$$

establishing 2 .

3. Write $N_{+}$for the complex subgroup of $N_{1}$ corresponding to the Lie algebra $\mathfrak{n}_{+}=\bigoplus_{\alpha \in \Sigma_{1}^{+}} \mathfrak{g}_{\mathbb{C}}^{\alpha}$. Notice that $N_{1}=N_{\mathbb{C}} \rtimes N_{+}$. Fix $g \in G$. Then $g$ can be uniquely expressed as

$$
g=n n_{+} a t u
$$

with $n \in N_{\mathbb{C}}, n_{+} \in N_{+}, a \in A, t \in \exp \left(i \mathfrak{t}_{1}\right)$ and $u \in U$. Replacing $g$ with $\tau(g)$ one obtains a decomposition

$$
\tau(g)=n^{\prime} n_{+}^{\prime} a^{\prime} t^{\prime} u^{\prime}
$$

for $n^{\prime} \in N_{\mathbb{C}}, n_{+}^{\prime} \in N_{+}, a^{\prime} \in A, t^{\prime} \in \exp \left(i \mathfrak{t}_{1}\right)$ and $u^{\prime} \in U$. We claim that $a=a^{\prime}$. Clearly this will prove the assertion in 3 .

We apply $\tau$ to $(4.3 .1)$

$$
\tau(g)=\tau(n) \tau\left(n_{+}\right) a t^{-1} \tau(u)
$$

With (4.3.2) we now get the equality

$$
n^{\prime} n_{+}^{\prime} a^{\prime} t^{\prime} u^{\prime}=\tau(n) \tau\left(n_{+}\right) a t^{-1} \tau(u)
$$

Observe that $\tau\left(N_{\mathbb{C}}\right)=N_{\mathbb{C}}$ and $\tau(U)=U$, but $\tau\left(N_{+}\right)=N_{-}$. Let $\theta$ denote the Cartan-involution on $G_{\mathbb{C}}$ with fixed point group $U$. Symmetrizing (4.3.3) we obtain that

$$
\begin{aligned}
\tau(g) \theta\left(\tau\left(g^{-1}\right)\right) & =n^{\prime} n_{+}^{\prime}\left(a^{\prime}\right)^{2}\left(t^{\prime}\right)^{2} \theta\left(n_{+}^{\prime}\right)^{-1} \theta\left(n^{\prime}\right)^{-1} \\
& =\tau(n) \tau\left(n_{+}\right) a^{2} t^{-2} \theta \tau\left(n_{+}\right)^{-1} \theta \tau(n)^{-1}
\end{aligned}
$$


Notice that $n_{+}^{\prime}\left(a^{\prime}\right)^{2}\left(t^{\prime}\right)^{2} \theta\left(n_{+}^{\prime}\right)^{-1}$ and $\tau\left(n_{+}\right) a^{2} t^{-2} \theta \tau\left(n_{+}\right)^{-1}$ belong to the reductive group $Z_{G_{\mathbb{C}}}(A)$. Thus the Bruhat-decomposition of $G_{\mathbb{C}}$ with respect to the parabolic subgroup $Z_{G_{\mathbb{C}}}(A) N_{\mathbb{C}}$ implies that $n^{\prime}=\tau(n)$. Hence we obtain the identity

$$
n_{+}^{\prime}\left(a^{\prime}\right)^{2}\left(t^{\prime}\right)^{2} \theta\left(n_{+}^{\prime}\right)^{-1}=\tau\left(n_{+}\right) a^{2} t^{-2} \theta \tau\left(n_{+}\right)^{-1}
$$

in $Z_{G_{\mathbb{C}}}(A)$. Notice that $A$ lies in the center of $Z_{G_{\mathbb{C}}}(A)$. Thus (4.3.4) implies that

$$
\left(a^{\prime}\right)^{2} x^{\prime}=a^{2} x \quad \text { for some } x^{\prime}, x \in\left[Z_{G_{\mathbb{C}}}(A), Z_{G_{\mathbb{C}}}(A)\right]_{0} .
$$

Hence $\left(a^{\prime}\right)^{2}=a^{2}$ and so $a=a^{\prime}$ as was to be shown.

Remark 4.3.2. (a) If $\mathfrak{m}=\mathfrak{t}_{1}$ is abelian, then the involution $\tau_{a}: M_{a} \rightarrow M_{a}$ is antisymplectic (cf. [8]). In fact, if $\mathfrak{m}$ is abelian, then $\tau\left(B_{1}\right)=B_{1}$ and $\mathbf{p r}_{\mathfrak{u}} \circ \tau=\tau \circ \mathbf{p r}_{\mathfrak{u}}$ holds. One verifies the anti-symplecticity of $\tau_{a}$ along the same lines as Lemma 4.3.1 1 .

(b) If $\mathfrak{m}$ is not abelian, then $\tau\left(B_{1}\right) \neq B_{1}$ and $\mathbf{p r}_{\mathfrak{u}} \circ \tau \neq \tau \circ \mathbf{p r}_{\mathfrak{u}}$. One can verify from (4.2.2) that $\tau_{a}$ is not anti-symplectic in this case (see Example 4.3.3 below). Mistakenly, as pointed out in [8], it was implicitly assumed in [13] that $\tau_{a}$ is always anti-symplectic.

Proof of Theorem 4.1.1. In view of Lemma 4.3.1 the assumptions of Theorem 3.1 are satisfied, and we can conclude that $\Phi\left(Q_{a}\right)=\Phi\left(M_{a}\right)=\operatorname{conv}\left(\operatorname{Fix}\left(M_{a}\right)\right)$. Standard structure theory shows that $\operatorname{Fix}\left(M_{a}\right)=\mathcal{W}$.a.

Example 4.3.3. We will show that $\tau_{a}$ is not anti-symplectic in case $\mathfrak{m}$ is not abelian. In this situation $\mathfrak{g}$ contains a subalgebra of type $\mathfrak{s o}(1,4)$. Therefore it is enough to consider the case of $G=\mathrm{SO}_{e}(1,4)$.

We start with some comments of general nature. First notice that every $n \in N$ is contained in some $M_{a}=\widetilde{b}(K a)$ for some $a \in A$. Now fix $n \in N$ and $a \in A$ such that $n \in M_{a}$. Let $X, Y \in i \mathfrak{p}$. Then $\tau_{a}(n)=n$ and

$$
d \tau_{a}(n) \widetilde{X}_{n}=-\widetilde{X}_{n} \text { and } \quad d \tau_{a}(n) \widetilde{Y}_{n}=-\widetilde{Y}_{n} .
$$

Thus if $\tau_{a}$ were anti-symplectic, then $\omega_{n}\left(\widetilde{X}_{n}, \widetilde{Y}_{n}\right)=0$. Below we will show that $\omega_{n}\left(\widetilde{X}_{n}, \widetilde{Y}_{n}\right) \neq 0$ for a specific choice of elements $n, X, Y$.

Now let $G=\mathrm{SO}_{e}(1,4)$. Then the Lie algebra of $G$ is given by

$$
\mathfrak{g}=\left\{\left(\begin{array}{cc}
0 & u^{t} \\
u & X
\end{array}\right): u \in \mathbb{R}^{4}, X \in \mathfrak{s o}(4)\right\} .
$$

The complexification of $\mathfrak{g}$ then is

$$
\mathfrak{g}_{\mathbb{C}}=\left\{\left(\begin{array}{cc}
0 & w^{t} \\
w & Z
\end{array}\right): w \in \mathbb{C}^{4}, Z \in \mathfrak{s o}(4, \mathbb{C})\right\} .
$$

Our choice of $\mathfrak{a}$ and $\mathfrak{t}_{1}$ will be

$$
\mathfrak{a}=\mathbb{R}\left(\begin{array}{ccccc}
0 & 0 & 0 & 0 & 1 \\
0 & 0 & 0 & 0 & 0 \\
0 & 0 & 0 & 0 & 0 \\
0 & 0 & 0 & 0 & 0 \\
1 & 0 & 0 & 0 & 0
\end{array}\right) \quad \text { and } \quad \mathfrak{t}_{1}=\mathbb{R}\left(\begin{array}{ccccc}
0 & 0 & 0 & 0 & 0 \\
0 & 0 & 1 & 0 & 0 \\
0 & -1 & 0 & 0 & 0 \\
0 & 0 & 0 & 0 & 0 \\
0 & 0 & 0 & 0 & 0
\end{array}\right)
$$


With appropriate $\Sigma_{1}^{+}$the nilpotent Lie algebras $\mathfrak{n}_{\mathbb{C}}$ and $\mathfrak{n}_{+}$are given by

$$
\mathfrak{n}_{\mathbb{C}}=\left\{\left(\begin{array}{ccc}
0 & z^{t} & 0 \\
z & 0 & -z \\
0 & z^{t} & 0
\end{array}\right): z \in \mathbb{C}^{3}\right\}
$$

and

$$
\mathfrak{n}_{1}=\mathbb{C}\left(\begin{array}{ccccc}
0 & 0 & 0 & 0 & 0 \\
0 & 0 & 0 & i & 0 \\
0 & 0 & 0 & 1 & 0 \\
0 & -i & -1 & 0 & 0 \\
0 & 0 & 0 & 0 & 0
\end{array}\right)
$$

Define an element $n \in N$ by

$$
n=\left(\begin{array}{ccccc}
\frac{5}{2} & 1 & 1 & 1 & -\frac{3}{2} \\
1 & 1 & 0 & 0 & -1 \\
1 & 0 & 1 & 0 & -1 \\
1 & 0 & 0 & 1 & -1 \\
\frac{3}{2} & 1 & 1 & 1 & -\frac{1}{2}
\end{array}\right) \in N
$$

Next we introduce elements $X, Y \in i \mathfrak{p}$ by

$$
X=\left(\begin{array}{ccccc}
0 & 0 & 0 & i & 0 \\
0 & 0 & 0 & 0 & 0 \\
0 & 0 & 0 & 0 & 0 \\
i & 0 & 0 & 0 & 0 \\
0 & 0 & 0 & 0 & 0
\end{array}\right) \text { and } Y=\left(\begin{array}{ccccc}
0 & i & 0 & 0 & 0 \\
i & 0 & 0 & 0 & 0 \\
0 & 0 & 0 & 0 & 0 \\
0 & 0 & 0 & 0 & 0 \\
0 & 0 & 0 & 0 & 0
\end{array}\right)
$$

A simple computation gives

$$
\operatorname{Ad}(n)^{-1} X=\left(\begin{array}{ccccc}
0 & -i & -i & \frac{3}{2} i & -i \\
-i & 0 & 0 & -i & i \\
-i & 0 & 0 & -i & i \\
\frac{3}{2} i & i & i & 0 & -\frac{1}{2} i \\
-i & -i & -i & \frac{1}{2} i & 0
\end{array}\right)
$$

and

$$
\operatorname{Ad}(n)^{-1} Y=\left(\begin{array}{ccccc}
0 & \frac{3}{2} i & -i & -i & -i \\
\frac{3}{2} i & 0 & i & i & -\frac{1}{2} i \\
-i & -i & 0 & 0 & i \\
-i & -i & 0 & 0 & i \\
-i & \frac{1}{2} i & -i & -i & 0
\end{array}\right)
$$

Then

$$
\mathbf{p r}_{\mathfrak{u}}\left(\operatorname{Ad}(n)^{-1} X\right)=\left(\begin{array}{ccccc}
0 & 0 & 0 & i & -i \\
0 & 0 & 0 & -1 & 0 \\
0 & 0 & 0 & 1 & 0 \\
i & 1 & -1 & 0 & 0 \\
-i & 0 & 0 & 0 & 0
\end{array}\right)
$$


Up to scalar we may identify B with the imaginary part of the trace form. With this normalization we then have

$$
\begin{aligned}
\omega_{n}\left(\widetilde{X}_{n}, \widetilde{Y}_{n}\right) & =\Im \operatorname{tr}\left(\left(\begin{array}{ccccc}
0 & 0 & 0 & i & -i \\
0 & 0 & 0 & -1 & 0 \\
0 & 0 & 0 & 1 & 0 \\
i & 1 & -1 & 0 & 0 \\
-i & 0 & 0 & 0 & 0
\end{array}\right) \cdot\left(\begin{array}{ccccc}
0 & \frac{3}{2} i & -i & -i & -i \\
\frac{3}{2} i & 0 & i & i & -\frac{1}{2} i \\
-i & -i & 0 & 0 & i \\
-i & -i & 0 & 0 & i \\
-i & \frac{1}{2} i & -i & -i & 0
\end{array}\right)\right) \\
& =\Im \operatorname{tr}\left(\begin{array}{ccccc}
0 & * & * & * & * \\
* & i & * & * & * \\
* & * & 0 & * & * \\
* & * & * & 1+i & * \\
* & * & * & * & -1
\end{array}\right)=2 \neq 0 .
\end{aligned}
$$

Following a suggestion from the referee we want to add a few remarks.

Remark 4.3.4. (a) Theorem 4.1.1 is the "real nonlinear" version of Kostant's convexity theorem. To complete its symplectic proof suggested in [13] we needed our generalized symplectic convexity theorem, Theorem 3.1. There is also a (real) linear version of Kostant's theorem: Let $Y \in \mathfrak{a}$. Then

$$
a(\operatorname{Ad}(K) . Y)=\operatorname{conv}(\mathcal{W} \cdot Y),
$$

where $\operatorname{conv}(\cdot)$ denotes the convex hull of $(\cdot)$ and $a: \mathfrak{g}=\mathfrak{k}+\mathfrak{a}+\mathfrak{n} \rightarrow \mathfrak{a}$ denotes the middle projection for the Iwasawa decomposition on the Lie algebra level.

In [4] and [13] symplectic proofs for this result have been given both for complex and for real $G$. In this case Duistermaat's theorem (Theorem 1.2 in the Introduction) suffices, because complex conjugation restricted to the coadjoint orbit $\operatorname{Ad}(K) . Y$ is antisymplectic with respect to the natural symplectic form on $\operatorname{Ad}(K) . Y$ for any complex semisimple group $G$.

(b) The linearization trick of Alekseev-Meinrenken-Woodward [1] (Chapter 3) can be generalized as follows. We adopt notation and numbering from [1].

Let $K$ be a compact connected Lie group equipped with an involutive automorphism $\sigma_{K}$ of the type described in Section 2.4 in [1. Suppose $M$ is a $K$-manifold with an involution $\sigma_{M}$. Let $\Omega, \omega$ be two forms on $M$, and $\Psi: M \rightarrow K^{*}, \Phi: M \rightarrow \mathfrak{k}^{*}$ related by (10) (i.e. equation (10) in [1]). Denote by $Q$ the fixed point set of $M$ under $\sigma_{M}$. Then the following are equivalent:

1. $(M, \Omega, \Psi)$ is a Hamiltonian $K$-space with $K^{*}$-valued moment map, and the involution $\sigma_{M}$ satisfies

(i) $\sigma_{M}(k \cdot m)=\sigma_{K}(k) \cdot\left(\sigma_{M}(m)\right) \quad \forall k \in K, m \in M$.

(ii) $\Psi \circ \sigma_{M}=\sigma_{K^{*}} \circ \Psi$.

(iii) $Q$ is a Lagrangian subspace of the symplectic manifold $(M, \Omega)$.

2. $(M, \omega, \Phi)$ is a Hamiltonian $K$-space with $\mathfrak{k}^{*}$-valued moment map, and the involution $\sigma_{M}$ satisfies

(i) $\sigma_{M}(k \cdot m)=\sigma_{K}(k) \cdot\left(\sigma_{M}(m)\right) \quad \forall k \in K, m \in M$.

(ii) $\Phi \circ \sigma_{M}=\sigma_{\mathfrak{k} *} \circ \Phi$.

(iii) $Q$ is a Lagrangian subspace of the symplectic manifold $(M, \omega)$.

This follows easily from (15) and (16).

Note that (i), (ii), (iii) become our properties (2.1.2), (2.1.3), (2.1.4) in the case $K=T$ and $\sigma_{K}(k)=k^{-1}$. 
Whereas linearization as in 3.1 in [1] can be generalized to a set-up with non-antisymplectic involutions, this is not clear for the results of 3.3 (and the corresponding statements in 3.4), since their proofs depend on the invariance of the flow $\widetilde{\varphi}^{s}$ under $\sigma_{M}$ in the isotopy Lemma 3.4.

(c) Duistermaat's generalized result on the tightness of Hamiltonian functions and its proof (Section 3 in [4]) also hold under the weaker conditions (2.1.2), (2.1.3), (2.1.4) on the involution $\tau$.

\section{REFERENCES}

[1] A. Alekseev, E. Meinrenken, C. Woodward, Linearization of Poisson actions and singular values of matrix products, Ann. Inst. Fourier 51, 6 (2001), 1691-1717. MR.1871286(2002j:53108)

[2] M.F. Atiyah, Convexity and commuting Hamiltonians, Bull. London Math. Soc. 14 (1) (1982), 1-15. MR0642416 (83e:53037)

[3] C. De Concini and C. Procesi, Quantum groups, Springer LNM 1565 (1992), 31-140. MR 1288995 (95j:17012)

[4] J.J. Duistermaat, Convexity and tightness for restrictions of Hamiltonian functions to fixed point sets of an antisymplectic involution, Trans. Amer. Math. Soc. 275 (1) (1983), 417-429. MR0678361 (84c:53035)

[5] V. Guillemin and S. Sternberg, Convexity properties of the moment mapping, Invent. Math. 67 (3) (1982), 491-513. MR0664117 (83m:58037)

[6] V. Guillemin and S. Sternberg, Symplectic techniques in physics, Cambridge University Press, 1990. MR1066693 (91d:58073)

[7] P. Heinzner and A. Huckleberry, Kählerian potentials and convexity properties of the momentum map., Invent. Math. 126 (1) (1996), 65-84. MR1408556 (98e:58075)

[8] J. Hilgert and K.-H. Neeb, Poisson Lie groups and non-linear convexity theorems, Math. Nachr. 191 (1998), 153-187. MR.1621294 (99k:58070)

[9] J. Hilgert, K.-H. Neeb and W. Plank, Symplectic Convexity Theorems and Coadjoint Orbits, Compositio Math. 94 (1994), 129-180. MR1302314 (96d:53053)

[10] F. Knop, Convexity of Hamiltonian manifolds, J. Lie Theory 12 (2) (2002), 571-582. MR1923787 (2003j:53131)

[11] B. Kostant, On convexity, the Weyl group and the Iwasawa decomposition, Ann. Sci. Ecole Norm. Sup. 6 (1973), 413-455. MR0364552 (51:806)

[12] K. Leichtweiß, Konvexe Mengen, Springer, 1979. MR0586235(81j:52001)

[13] J.-H. Lu and T. Ratiu, On the nonlinear convexity theorem of Kostant, J. Amer. Math. Soc. 4 (2) (1991), 349-363. MR1086967 (92a:58048)

[14] R. Sjamaar, Convexity Properties of the Moment Mapping Re-examined, Advances in Math. 138 (1998), 46-91. MR.1645052(2000a:53148)

Department of Mathematics, University of Oregon, Eugene, Oregon 97403-1221

E-mail address: kroetz@math.uoregon.edu

Department of Mathematics, Ohio State University, 231 West 18th Avenue, ColumBus, Оніо

E-mail address: otto@math.ohio-state.edu 